\title{
A measurement method of knee joint space width by ultrasound: a large multicenter study
}

\author{
Jiaan Zhu ${ }^{1}$, Bing $\mathrm{Li}^{1}$, Li Qiu ${ }^{2}$, Hongmei Liu ${ }^{3}$, Mi Zhang ${ }^{1}$, Yuexiang Wang ${ }^{4}$, Ping Wang ${ }^{5}$, Dan Jiao ${ }^{6}$, Tao Chen ${ }^{7}$, \\ Xueling Liu ${ }^{8}$, Ligang Cui ${ }^{9}$, Yong Shan ${ }^{10}$, Baoming Luo ${ }^{11}$, Ning Lin ${ }^{12}$, Xing Hua ${ }^{13}$, Zhenlong Hu ${ }^{14}$, \\ Yue $\mathrm{Hu}^{1}$, Bin Tu${ }^{15}$, Yuanyi Zheng ${ }^{16}$, Shuqiang $\mathrm{Chen}^{17}$, Shiliang $\mathrm{Xu}^{18}$, Jianying Mao ${ }^{19}$, Weiyong Liu ${ }^{20}$, \\ Minghui Xiang ${ }^{21}$, Jia Lii ${ }^{22}$, Jian Chen ${ }^{23}$, Yuanjiao Tang ${ }^{2}$, Siming Chen ${ }^{4}$, Yanni He ${ }^{3}$, Ting Dai ${ }^{6}$, \\ Shumin Zhang ${ }^{7}$, Yuanyuan Zhang ${ }^{9}$, Mingdi Fang ${ }^{10}$, Shaoyun Hao ${ }^{11}$, Xiaoyan Lin ${ }^{12}$, Xiuzhen $\mathrm{He}^{15}$, \\ Bo Bao ${ }^{18}$, Zhanguo $\mathrm{Xi}^{24}$, Xiaojing Peng ${ }^{25}$, Qunxia Zhang ${ }^{26}$, Guoqing $\mathrm{Du}^{27}$
}

${ }^{1}$ Department of Ultrasound, Peking University People's Hospital, Beijing 100044, China; ${ }^{2}$ Department of Ultrasound, Sichuan University West China Hospital, Chengdu 610041, China; ${ }^{3}$ Department of Ultrasound, Guangdong Second Provincial General Hospital, Guangzhou 510317, China; ${ }^{4}$ Department of Ultrasound, Chinese People's Liberation Army General Hospital, Beijing 100853, China; ${ }^{5}$ Department of Ultrasound, Third Affiliated Hospital of Southern Medical University, Guangzhou 510000, China; ${ }^{6}$ Department of Ultrasound, China-Japan Union Hospital of Jilin University, Changchun 130033, China; ${ }^{7}$ Department of Ultrasound, Beijing Jishuitan Hospital, Beijing 100009, China; ${ }^{8}$ Department of Ultrasound, The First Affiliated Hospital of Guangxi University of Chinese Medicine, Nanning 530023, China; ${ }^{9}$ Department of Ultrasound, Peking University Third Hospital, Beijing 100191, China; ${ }^{10}$ Department of Ultrasound, The Second Hospital of Anhui Medical University, Hefei 230601, China; ${ }^{11}$ Department of Ultrasound, Sun Yat-Sen Memorial Hospital, Sun Yat-Sen University, Guangzhou 510120, China; ${ }^{12}$ Department of Ultrasound, Fujian Provincial Hospital, Fuzhou 350001, China; ${ }^{13}$ Department of Ultrasound, The First Hospital Affiliated to Army Medical University (Southwest Hospital), Chongqing 400038, China; ${ }^{14}$ Department of Ultrasound, Shanghai Jiao Tong University Affiliated First People's Hospital, Shanghai 210620, China; ${ }^{15}$ Department of Ultrasound, Foshan Hospital of Traditional Chinese Medicine, Foshan 528000, China; ${ }^{16}$ Department of Ultrasound, Shanghai Jiao Tong University Affiliated Sixth People's Hospital, Shanghai 200233, China; ${ }^{17}$ Department of Ultrasound, The First Affiliated Hospital of Fujian Medical University, Fuzhou 350005, China; ${ }^{18}$ Department of Ultrasound, Haikou People's Hospital, Haikou 570208, China; ${ }^{19}$ Department of Ultrasound, Shanghai Guanghua Hospital of Integrated Traditional Chinese and Western Medicine, Shanghai 200052, China; ${ }^{20}$ Department of Ultrasound, The First Affiliated Hospital of University of Science and Technology of China, Hefei 230036, China; ${ }^{21}$ Department of Ultrasound, Central Hospital Affiliated to Shenyang Medical College, Shenyang 110027, China; ${ }^{22}$ Department of Ultrasound, Zhongda Hospital Southeast University, Nanjing 210009, China; ${ }^{23}$ Department of Ultrasound, Yan'an Hospital of Kunming City, Kunming 650051 , China; ${ }^{24}$ Department of Ultrasound, Luoyang Orthopedic-Traumatological Hospital of Henan Province, Luoyang 471000, China; ${ }^{25}$ Department of Ultrasound, Jiangsu Province Hospital, Nanjing 210029, China; ${ }^{26}$ Department of Ultrasound, the Second Affiliated Hospital of Chongqing Medical University, Chongqing 400065, China; ${ }^{27}$ Department of Ultrasound, the Second Affiliated Hospital of Harbin Medical University, Harbin 150001, China

Correspondence to: Jiaan Zhu. Department of Ultrasound, Peking University People's Hospital, No. 11 Xizhimen South Street, Xicheng District, Beijing 100044, China. Email: zhujiaan@pkuph.edu.cn.

Background: Although plain radiology is the primary method for assessing joint space width (JSW), it has poor sensitivity to change over time in regards to determining longitudinal progression. We, therefore, developed a new ultrasound (US) measurement method of knee JSW and aimed to provide a monitoring method for the change of JSW in the future.

Methods: A multicenter study was promoted by the Professional Committee of Musculoskeletal Ultrasound, the Ultrasound Society, and the Chinese Medical Doctor Association. US study of knee specimens determined the landmarks for ultrasonic measurement of knee JSW. The US of 1,272 participants from 27 centers was performed to discuss the feasibility and possible influencing factors of knee JSW. The landmarks for US measurement of knee JS, the inflection point of medial femoral epicondyle and the proximal end of the tibia, were determined.

Results: The mean knee JSW1 (medial knee JSW) was $8.57 \pm 1.95 \mathrm{~mm}$ in females and $9.52 \pm 2.31 \mathrm{~mm}$ in males. The mean knee JSW2 (the near medial knee JSW) was $9.07 \pm 2.24 \mathrm{~mm}$ in females and $10.17 \pm 2.35 \mathrm{~mm}$ in males. 
The JSW values of males were significantly higher than those of females, with a statistical difference. JSW values were negatively correlated with age and body mass index (BMI) to different degrees and positively correlated with height.

Conclusions: The novel US measurement method can be used to measure knee JSW.

Keywords: Ultrasound (US); joint space width (JSW); knee

Submitted Feb 29, 2020. Accepted for publication Apr 09, 2020.

doi: 10.21037/qims-20-373

View this article at: http://dx.doi.org/10.21037/qims-20-373

\section{Introduction}

Knee joint space narrowing (JSN) can be seen in patients with distinct rheumatic diseases and is also a critical parameter that could be affected by disease-modifying therapy $(1,2)$. Despite technological advances and the availability of magnetic resonance imaging (MRI) and ultrasound (US) modalities, plain radiography is still the primary method for assessing knee joint structural damage. The measurement of radiographic joint space width (JSW) is the most commonly proposed and widely used method of assessing JSN $(1,3)$. Once the diagnosis of arthritis is made, it is necessary to assess the changes of JSW while the patients are followed up or undergo medication. However, due to the slight changes of JSW in these periods $(1,3,4)$, the reduced responsiveness to change over time is a limitation in determining longitudinal progression $(5,6)$. Hence, the patients may not only receive insufficient diagnostic benefits from plain radiography but also be subjected to radiation exposure.

Significant advances have been made within the field of imaging in distinct arthritis over the past decade $(7,8)$. US has been increasingly used in recent years as a technique to evaluate, diagnose, and monitor osteoarthritis and rheumatoid arthritis (9-11). However, few reports have described the scientific nature of US as a measurement method of JSW. The European League Against Rheumatism (EULAR) task force was, therefore, convened to develop evidence-based recommendations on the use of imaging of the joints in the clinical management of rheumatoid arthritis (10). In light of the importance of JSW for functional status (2), specific reference to JSN has been made by the EULAR task force in the proposed future research agenda, purpose of which is to further assess the importance of imaging, especially of MRI and US in the evaluation of JSN and cartilage loss. Osteoarthritis or rheumatoid arthritis commonly affect the knee. US has the advantage of causing no radiation and facilitating convenient follow-up. Therefore, the goal of the present study was to develop a novel US measurement method of knee JSW, with the hope of providing a future monitoring method for the change of JSW.

\section{Methods}

\section{Participants}

This prospective, multicenter, observational study was supported by the Professional Committee of Musculoskeletal Ultrasound, the Ultrasound Society, and the Chinese Medical Doctor Association, and studied subjects from 27 centers between October 2018 and March 2019 (ChiCTR1800020043). All the examiners were from these 27 hospitals and had 2-3 years or more of experience in musculoskeletal US. Participants were mainly patients or their family members who visited the US department and participated in the physical examination. Through detailed consultation and physical examination, people deemed unsuitable for the study were excluded according to the exclusion criteria. In total, 1,272 participants with no knee symptoms or other musculoskeletal complaint, and ranging in age from 18 to 84 years old, were recruited from these 27 centers.

The exclusion criteria were as follows: (I) knee deformity (according to the diagnostic criteria of International Classification of Disease-10-); (II) rheumatic diseases; (III) metabolic disorder known to affect bone metabolism; (IV) inflammatory disease or other medical condition affecting the knee joint; (V) history of knee surgery or trauma; (VI) intra-articular injection of the knee; (VII) medication influencing bone (steroids, vitamin $\mathrm{D}$, or calcium intake); (VIII) long-term weight-bearing or other work that may cause knee injury; (IX) knee pain within the past year. All participants provided their written, informed consent, and 

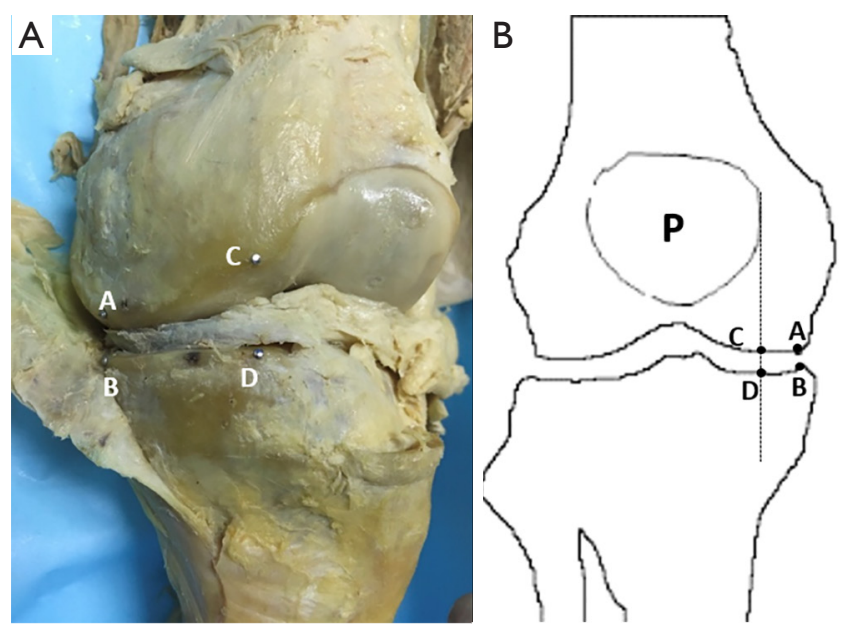

Figure 1 Photograph of knee specimen. (A) The 4 nails were inserted at point $\mathrm{A}, \mathrm{B}, \mathrm{C}$, and $\mathrm{D}$, respectively, and were used as landmarks for ultrasonic measurement; (B) schematic diagram of the measurement location.
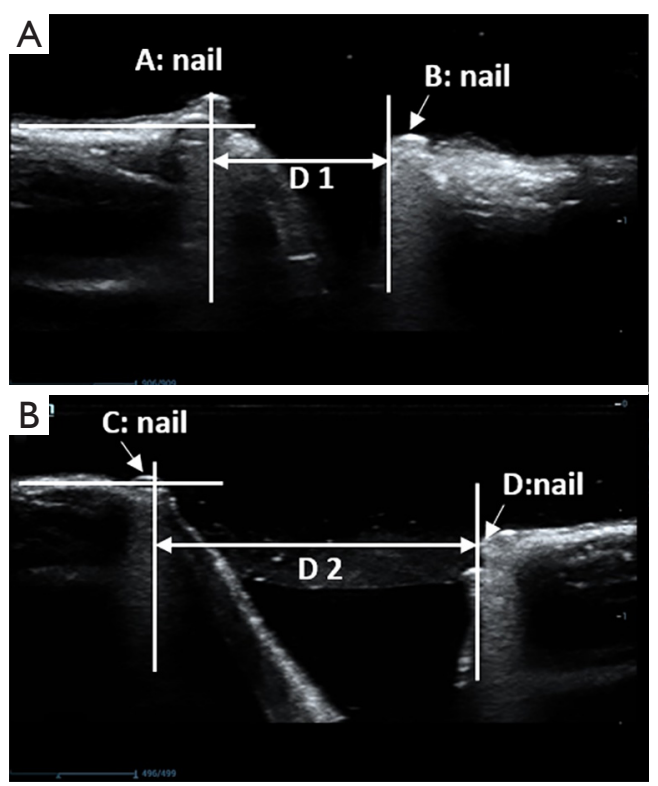

Figure 2 The location of points A, B, C, and D on the sonogram.

the study was conducted with the approval of the ethics committees of Peking University People's Hospital.

\section{Ultrasonography of cadaveric specimens}

To observe the relationship between the position detected by US and the exact position of the human body, we studied

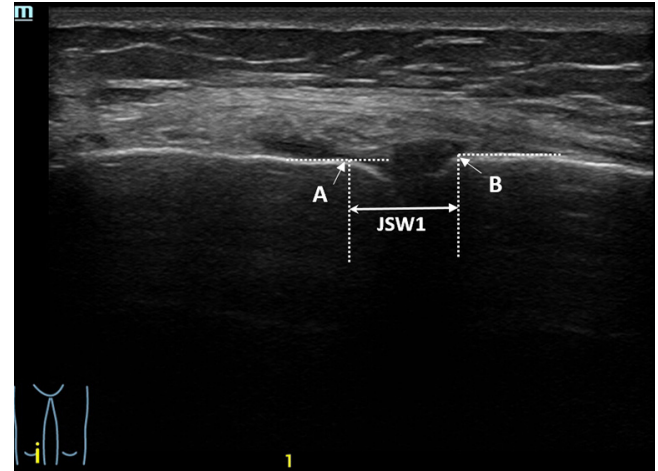

Figure 3 Measurement of medial joint space width (JSW1) at coronal scan.

4 formalin-fixed knees from the Department of Anatomy, Peking University Health Science Center (2 right knees and 2 left knees) to determine the landmarks for ultrasonic measurement of knee JSW. After the medial side of the knee was dissected, the cartilage margin at the medial femoral epicondyle was defined as point $\mathrm{A}$, and the cartilage margin close to the tibia was defined as the point $\mathrm{B}$. We drew a vertical line along the medial edge of the patella, which intersected the lower edge of the femur as point $\mathrm{C}$ and the upper edge of the tibia as point $\mathrm{D}$. We inserted a nail at points $\mathrm{A}, \mathrm{B}, \mathrm{C}$, and D (Figure 1). Then, US coronal scanning identified point $\mathrm{A}$ and point $\mathrm{B}$, and sagittal scanning identified point $\mathrm{C}$ and point $\mathrm{D}$.

The location of point $A$ or point $C$ on the sonogram showed the inflection point of the medial femoral epicondyle. A line was drawn along the distal femur, and the location that just deviated from the straight line was point A or C (Figure 2). The location of point B or D was at the proximal end of the tibia. Point A showed the inflection point of the lateral condyle on the sonogram.

\section{Ultrasonography of participants}

All subjects had a US examination of the bilateral knee joint using different machines with a 7-15 MHz linear transducer (Mindray Resona 7, Toshiba Aplio 500, and GE Logiq E9). After a coronal scan showed the long-axis of the medial collateral ligament, and with the participants in a supine position with their legs relaxing naturally, point A and point $\mathrm{B}$ were identified, and the medial JSW (JSW1) was measured (Figure 3). Then, the transducer moved to the medial edge of the patella, sagittal scan identified point $\mathrm{C}$ and point $\mathrm{D}$, and the near medial JSW (JSW2) was 
measured (Figure 4; Videos 1,2).

After the data were collected, all knee sonograms were read and judged again at Peking University People's Hospital.

The calculation of the intra-class correlation coefficient (ICC) had 2 parts. The first part occurred before the examination. After the joint learning session, 2 randomly selected physicians (from different hospitals) inspected the same 10 volunteers attending the session. The ICC was 0.92 , which was calculated between the 2 doctors. The next day, 1 of the 2 doctors reexamined the same volunteers. The ICC was 0.95 , which was calculated of the same person at different time. Both of the ICCs were above 0.9.

The second part occurred at the image-reading stage. Two examiners were randomly selected from the random sampling center. Both of them had at least 2-3 years of career experience. The images of 10 patients were read by them. The ICC was 0.98 , which was calculated between the two examiners. Ten days later, 1 of the 2 examiners reread the same images of the 10 patients. The ICC was 0.99 , which was calculated of the same examiner at different time.

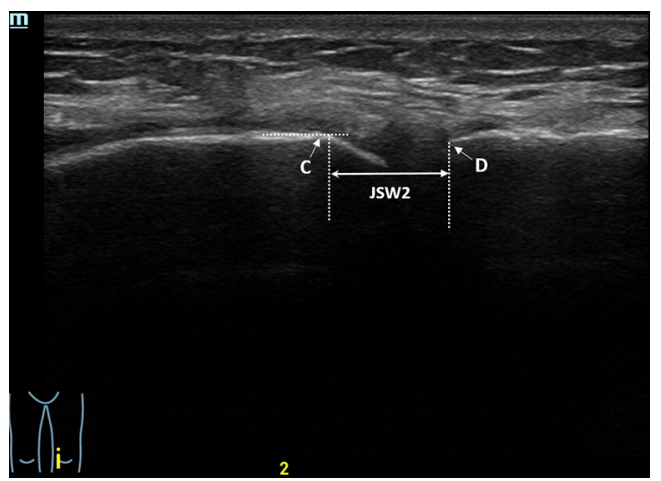

Figure 4 Measurement of the near middle joint space width (JSW2) at sagittal scan.

\section{Statistical analysis}

Mean differences in joint measurements between the sexes were examined using independent sample $t$-tests. The relationship between joint space and body size parameters were examined in the sex-specific samples using bivariate parametric correlation procedures (Pearson productmoment correlation).

Stepwise multivariate linear regression was used to identify those variables that significantly influenced joint space. Statistical significance was determined as a $\mathrm{P}$ value $<0.05$. All statistical procedures were performed using the SPSS platform, version 21.0 (SPSS Inc., Chicago, IL, USA).

\section{Results}

After excluding cases for being osteophytes or having incomplete data due to restricted information access, 900 of 1,272 subjects were enrolled in the statistical study. Table S1 shows the number of study subjects in each hospital. The mean knee JSW1 was $8.57 \pm 1.95 \mathrm{~mm}$ in females and $9.52 \pm 2.31 \mathrm{~mm}$ in males. The mean knee JSW2 was $9.07 \pm 2.24 \mathrm{~mm}$ in females and $10.17 \pm 2.35 \mathrm{~mm}$ in males. Table 1 shows the descriptive statistics for age and body size data. The measurement results of the knee JSW are presented in Table 2, while the results of the sex-specific correlation analyses are presented in Table 3. The scatter plot showed the relationship between JSW and age. Varying by gender, JSW tended to decrease with the increase of age (Figure 5). Table 4 shows the influence of sex, height, weight, and BMI on knee JSW.

\section{Discussion}

In the present study, a novel US measurement method of JSW of the knee was developed. However, the main goal was to provide a future method for monitoring the

Table 1 Descriptive characteristics for participants

\begin{tabular}{|c|c|c|c|c|c|}
\hline Characteristic & \multicolumn{2}{|c|}{ Female $(n=462)$} & \multicolumn{2}{|c|}{ Male $(n=438)$} & $P$ value \\
\hline Age (years) & 47.96 & 14.82 & 48.05 & 16.26 & 0.09 \\
\hline Height (cm) & 159.06 & 5.44 & 170.17 & 6.27 & $<0.01$ \\
\hline Weight (kg) & 57.73 & 8.13 & 70.01 & 11.07 & $<0.01$ \\
\hline
\end{tabular}

Study sample characteristics with associated P values from independent sample $t$-tests. SD, standard deviation; BMI, body mass index. 
Table 2 The results of knee joint space width measurement

\begin{tabular}{|c|c|c|c|c|c|}
\hline Knee joint space width & \multicolumn{2}{|c|}{ Female $(n=462)$} & \multicolumn{2}{|c|}{ Male $(n=438)$} & $P$ value \\
\hline Left JSW1 (mm) & 8.42 & 1.82 & 9.38 & 2.17 & $<0.01$ \\
\hline Left JSW2 (mm) & 9.18 & 2.24 & 10.28 & 2.38 & $<0.01$ \\
\hline Right JSW1 (mm) & 8.71 & 2.06 & 9.65 & 2.43 & $<0.01$ \\
\hline
\end{tabular}

JSW, joint space width; JSW1, medial knee joint space width; JSW2, near medial knee joint space width; SD, standard deviation.

Table 3 Sex-specific correlation analyses of knee joint space width

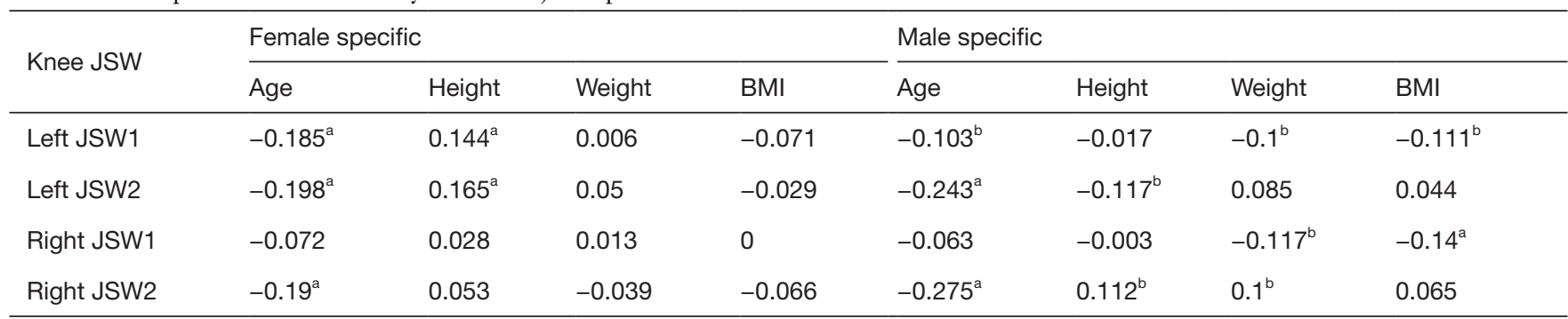

${ }^{a}$, correlation is significant at the 0.01 level; ${ }^{b}$, correlation is significant at the 0.05 level. JSW, joint space width; JSW1, medial knee joint space width; JSW2, near medial knee joint space width.

change of JSW in the future rather than diagnosis JSN. Although plain radiology is the primary method for assessing joint structural damage, it has poor sensitivity to change over time in regards to determining longitudinal progression (3-5). JSW changes slightly as joint diseases progress. A systematic review reported that the annual rate of medial JSN was $0.13 \pm 0.15 \mathrm{~mm} /$ year (1). Nevitt's study on the natural course of knee osteoarthritis showed a joint space loss of $0.24 \pm 0.59 \mathrm{~mm}$ every 3 years (12). Usually, it is difficult to capture such subtle changes in routine clinical practice. For instance, patients with the rheumatic disease may need short-term follow-up for different reasons (e.g., unexpected rapid progression of symptoms, clinical pharmaceutical trial, etc.). In these cases, not only do patients not receive adequate diagnoses from plain radiography, but they are also exposed to unnecessary radiation exposure. Some researchers have even suggested that the detection of JSN on radiography seems outdated because of the shortcomings of plain radiology which include insensitivity to change, non-specificity, and absence of reproducibility in longitudinal studies (6). There are several advantages of the US, which include its lack of ionizing radiation, higher resolution compared to plain radiography, and quick-to-perform nature (13). Thus, the evaluating the changes of JSW by US may be beneficial in clinical practice.

Yanagisawa et al. (14) reported a measurement method of knee JSW using US, where JSW was measured as the distance from the peripheral femoral cortices to the peripheral tibial cortices. However, because of the curved medial femur epicondyle, the medial edge of the tibia and the medial edge of the medial femoral condyle are not always on the same line on the sonogram.

It is crucial to accurately identify the measurement points before the JSW calculation is performed, which directly influences the JSW calculation results. In the present study, we defined the 4 relatively stable measuring points. Although the measurement locations were not at the center of the knee JSW, we aimed to apply the track changes in JSW, that is, to observe the changes of measurements at the same location. The main reason that we measured knee JSW in 2 places was that there may be overlying osteophytes in the medial knee that obscured the acoustic window and prevented visualization of the measurement joint. In the present study, we only measured 2 locations for medial knee JSW. The main reason for this is that the progression of JSN is more common in the medial than in the lateral compartment (15). The change of medial 

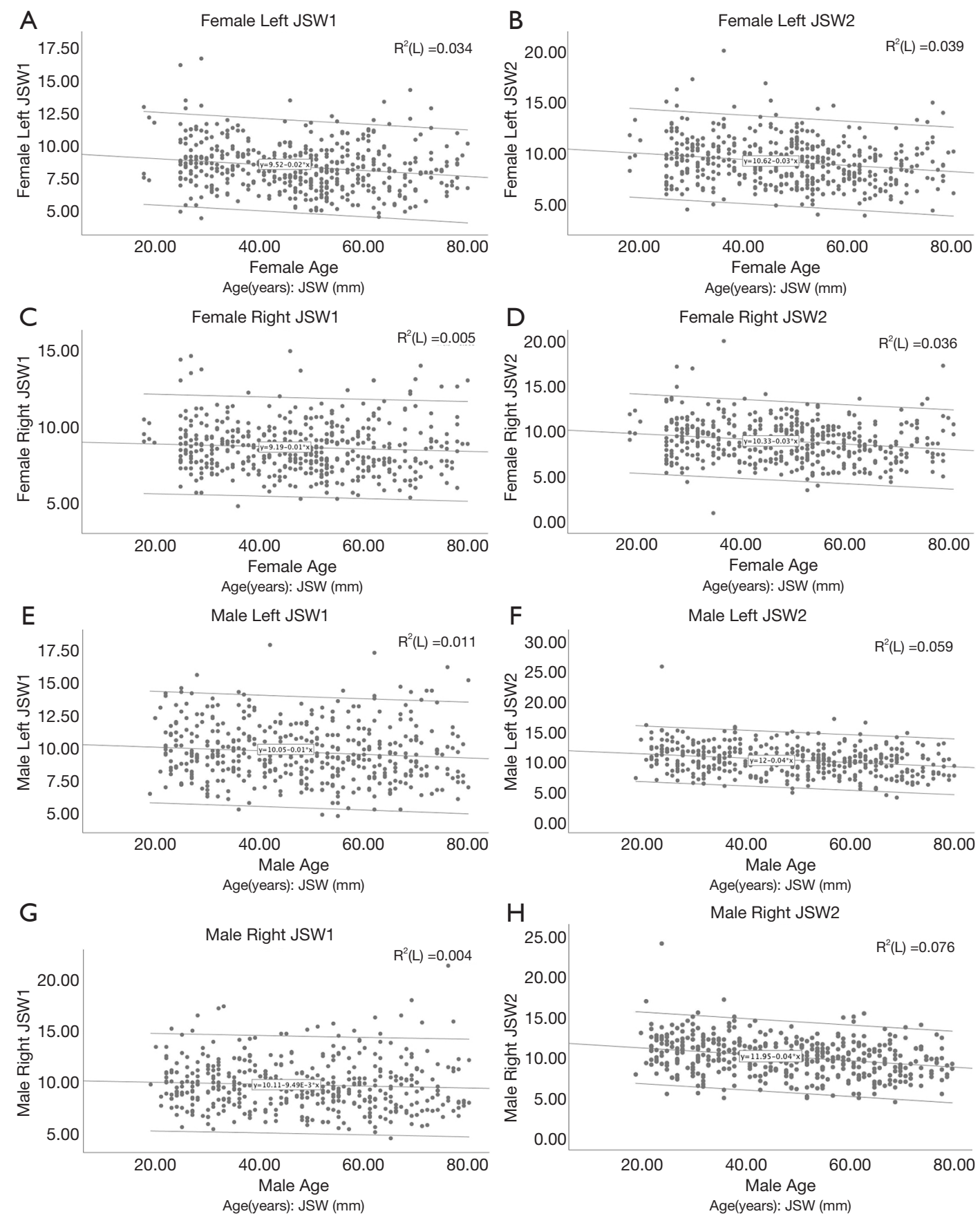

Figure 5 Scatter plots divided by gender. The horizontal axis is age, and the vertical axis is the value of joint space. When combined with data from Table 3, the distribution of JSW changes with age can be discerned. The line in the middle is the fitting straight line, and the line on either side is the $95 \%$ confidence interval (the $\mathrm{P}$ values from A to $\mathrm{H}$ follow respectively: A, 0.000; $\mathrm{B}, 0.000 ; \mathrm{C}, 0.121 ; \mathrm{D}, 0.000 ; \mathrm{E}, 0.030$; F, 0.000; G, 0.186; H, 0.000). (A) Female left JSW1; (B) Female left JSW2; (C) Female right JSW1; (D) Female right JSW2; (E) Male left JSW1; (F) Male left JSW2; (G) Male right JSW1; (H) Male right JSW2. JSW1, medial knee joint space width; JSW2, near medial knee joint space width. 
Table 4 The influence of sex, height, weight, and BMI on knee joint space width

\begin{tabular}{|c|c|c|c|c|c|}
\hline Model & Independent variable & Coefficients & $\mathrm{t}$ & $P$ value & $\mathrm{R}^{2}$ \\
\hline \multicolumn{6}{|l|}{ Left JSW1 } \\
\hline \multirow[t]{3}{*}{ Final model } & Constant & 9.288 & 41.431 & 0.000 & 0.073 \\
\hline & Sex & 0.961 & 7.267 & 0.000 & \\
\hline & Age & -0.018 & -4.222 & 0.000 & \\
\hline \multirow[t]{4}{*}{ Final model } & Constant & 4.926 & 2.260 & 0.024 & 0.108 \\
\hline & Sex & 0.714 & 3.394 & 0.001 & \\
\hline & Age & -0.030 & -5.943 & 0.000 & \\
\hline & Height & 0.036 & 2.696 & 0.007 & \\
\hline \multirow{2}{*}{ Final model } & Sex & 1.012 & 6.606 & 0.000 & \\
\hline & BMI & -0.054 & -2.263 & 0.024 & \\
\hline \multicolumn{6}{|l|}{ Right JSW2 } \\
\hline \multirow[t]{3}{*}{ Final model } & Constant & 10.603 & 42.409 & 0.000 & 0.108 \\
\hline & Sex & 1.112 & 0.148 & 0.000 & \\
\hline & Age & -0.034 & 0.005 & 0.000 & \\
\hline
\end{tabular}

In these analyses, L-JSW1, L-JSW2, R-JSW1, R-JSW2 served as the dependent variable, and sex, height, weight, and BMI were independent variables. In the analysis of L-JSW1, the first model identified sex and age as the best predictor, height, weight and BMI were excluded from the final model. In the final two-term model (sex, age), 7.3\% of the variance in L-JSW1 was explained. Similarly, L-JSW2, R-JSW1 and R-JSW2 were 10.8\%, 4.7\%, 10.8\%. JSW, joint space width; JSW1, medial knee joint space width; JSW2, near medial knee joint space width; L, left; R, right; BMI, body mass index.

knee JSW has been recommended as the primary measure of biological effect in osteoarthritis by expert consensus (16). Additionally, the study showed that the measurement of location-specific JSW in detecting osteoarthritis progression seemed to be superior to minimum JSW assessment regarding the prediction of progression (17).

Many factors affect the reproducibility of knee JSW measurements, such as subject positioning, US scanning procedure, site of measurement, measuring methods, and the readers. In this study, attempts have been made to increase the reproducibility of JSW by standardizing US scanning procedures. These attempts primarily focused on the site of measurement. By repeatedly observing the structure of the knee joint, we defined the medial positions, which made it easier to obtain standardized measurement data.

Older people may have degenerative arthritis, and our participants are asymptomatic with no visible joint deformity and no clear history of osteoarthritis in the past. As the embodiment of the people in the process of natural non-pathological conditions, part of the older participants' US showed osteophyte after inspection, after agreeing to the $\mathrm{X}$-ray, revealed a JSN. We excluded this part in the statistics, so results show the absence of a typical degenerative joint disease state of joint clearance. Of course, the probability of old bone arthritis is higher, and there may be part of is not apparent, but those that had very early lesions were included in the study. We considered this part of being an acceptable physiological change.

The present study has several limitations. Firstly, the measurements of JSW are not the smallest gaps (the central part of the joint) in the knee joint. We developed the US measurement method to anticipate the change of JSW in the future rather than as a diagnosis of JSN. Future work should be performed to prove the validity of this method for documenting change over time in the knee osteoarthritis 
or rheumatic arthritis. Secondly, there was no attempt to correlate US measurements of JSW with the reference standard of JSW measurements on knee radiographs. The main reason was that not all the subjects were willing to accept the X-ray examination. Also, the 2 methods did not measure the same location. Even more importantly, this method of US measurement is designed for the long-term follow-up of knee JSW. Thirdly, we only measured JSW in a supine position with the legs naturally relaxed. Further studies are needed to strengthen our study.

In conclusion, the present study determined the landmarks for the US measurement of knee JSW and may provide a novel method for monitoring the change of JSW in the future.

\section{Acknowledgments}

We would like to thank Huabin Zhang, Zhilan Zhang, Junping Zhu, Lini Xian, Yumei Kuang, Qiuting Ren, Jie Chen, Lei Ye, Jinjun Shi, Yaxin Qiao, Dan Wang, Yun He, Yinan Huang, Fan Xiang, Wenhong Yi, Jiarui Du, Yibing Zhao, Haijie Wang, Shan Cheng, and Zhou Chen for their help in recruiting subjects.

Funding: This work was supported in part by the National Natural Science Foundation of China (No. 81571684).

\section{Footnote}

Conflicts of Interest: All authors have completed the ICMJE uniform disclosure form (available at http://dx.doi. org/10.21037/qims-20-373). The authors have no conflicts of interest to declare.

Ethical Statement: The study was approved by the Ethics Committee of Peking University People's Hospital (NO.:2015PHB206-01). Written informed consent was obtained from all patients for publication of this study and any accompanying images. A copy of the written consent is available for review by the editor-in-chief of this journal.

Open Access Statement: This is an Open Access article distributed in accordance with the Creative Commons Attribution-NonCommercial-NoDerivs 4.0 International License (CC BY-NC-ND 4.0), which permits the noncommercial replication and distribution of the article with the strict proviso that no changes or edits are made and the original work is properly cited (including links to both the formal publication through the relevant DOI and the license).
See: https://creativecommons.org/licenses/by-nc-nd/4.0/.

\section{References}

1. Emrani PS, Katz JN, Kessler CL, Reichmann WM, Wright EA, McAlindon TE, Losina E. Joint space narrowing and Kellgren-Lawrence progression in knee osteoarthritis: an analytic literature synthesis. Osteoarthritis Cartilage 2008;16:873-82.

2. Lillegraven S, van der Heijde D, Uhlig T, Kvien TK, Haavardsholm EA. What is the clinical relevance of erosions and joint space narrowing in RA? Nat Rev Rheumatol 2012;8:117-20.

3. Platten M, Kisten Y, Kälvesten J, Arnaud L, Forslind K, van Vollenhoven R. Fully automated joint space width measurement and digital $\mathrm{X}$-ray radiogrammetry in early RA. RMD Open 2017;3:e000369.

4. Reichmann WM, Maillefert JF, Hunter DJ, Katz JN, Conaghan PG, Losina E. Responsiveness to change and reliability of measurement of radiographic joint space width in osteoarthritis of the knee: a systematic review. Osteoarthritis Cartilage 2011;19:550-6.

5. Hafezi-Nejad N, Guermazi A, Demehri S, Roemer FW. New imaging modalities to predict and evaluate osteoarthritis progression. Best Pract Res Clin Rheumatol 2017;31:688-704.

6. Guermazi A, Roemer FW, Burstein D, Hayashi D. Why radiography should no longer be considered a surrogate outcome measure for longitudinal assessment of cartilage in knee osteoarthritis. Arthritis Res Ther 2011;13:247.

7. Østergaard M, Pedersen SJ, Døhn UM. Imaging in rheumatoid arthritis--status and recent advances for magnetic resonance imaging, ultrasonography, computed tomography and conventional radiography. Best Pract Res Clin Rheumatol 2008;22:1019-44.

8. Nieminen MT, Casula V, Nevalainen MT, Saarakkala S. Osteoarthritis year in review 2018: imaging. Osteoarthritis Cartilage 2019;27:401-11.

9. Sakellariou G, Conaghan PG, Zhang W, et al. EULAR recommendations for the use of imaging in the clinical management of peripheral joint osteoarthritis. Ann Rheum Dis 2017;76:1484-94.

10. Colebatch AN, Edwards CJ, Østergaard M, et al. EULAR recommendations for the use of imaging of the joints in the clinical management of rheumatoid arthritis. Ann Rheum Dis 2013;72:804-14.

11. Ren J, Zhu J, Li D, Li W, Liu F. The value of contrastenhanced ultrasonography to detect the sacroiliac joint 
for predicting relapse after discontinuation of antitumor necrosis factor therapy in patients with ankylosing spondylitis. Quant Imaging Med Surg 2019;9:1110-7.

12. Nevitt MC, Peterfy C, Guermazi A, Felson DT, Duryea J, Woodworth T, Chen H, Kwoh K, Harris TB. Longitudinal performance evaluation and validation of fixed-flexion radiography of the knee for detection of joint space loss. Arthritis Rheum 2007;56:1512-20.

13. Hayashi D, Roemer FW, Guermazi A. Recent advances in research imaging of osteoarthritis with focus on MRI, ultrasound and hybrid imaging. Clin Exp Rheumatol 2018;36 Suppl 114:43-52.

14. Yanagisawa S, Ohsawa T, Saito K, Kobayashi T, Yamamoto A, Takagishi K. Morphological evaluation and diagnosis of medial type osteoarthritis of the knee using ultrasound. J Orthop Sci 2014;19:270-4.

15. Ledingham J, Regan M, Jones A, Doherty M. Factors

Cite this article as: Zhu J, Li B, Qiu L, Liu H, Zhang M, Wang Y, Wang P, Jiao D, Chen T, Liu X, Cui L, Shan Y, Luo B, Lin N, Hua X, Hu Z, Hu Y, Tu B, Zheng Y, Chen S, Xu S, Mao J, Liu W, Xiang M, Li J, Chen J, Tang Y, Chen S, He Y, Dai T, Zhang S, Zhang Y, Fang M, Hao S, Lin X, He X, Bao B, Xi Z, Peng X, Zhang Q, Du G. A measurement method of knee joint space width by ultrasound: a large multicenter study. Quant Imaging Med Surg 2020;10(5):979-987. doi: 10.21037/ qims-20-373 affecting radiographic progression of knee osteoarthritis. Ann Rheum Dis 1995;54:53-8.

16. Altman R, Brandt K, Hochberg M, Moskowitz R, Bellamy N, Bloch DA, Buckwalter J, Dougados M, Ehrlich G, Lequesne M, Lohmander S, Murphy WA Jr, Rosario-Jansen T, Schwartz B, Trippel S. Design and conduct of clinical trials in patients with osteoarthritis: recommendations from a task force of the Osteoarthritis Research Society. Results from a workshop. Osteoarthritis Cartilage 1996;4:217-43.

17. Duryea J, Neumann G, Niu J, Totterman S, Tamez J, Dabrowski C, Le Graverand MP, Luchi M, Beals CR, Hunter DJ. Comparison of radiographic joint space width with magnetic resonance imaging cartilage morphometry: analysis of longitudinal data from the Osteoarthritis Initiative. Arthritis Care Res (Hoboken) 2010;62:932-7. 
Supplementary

Table S1 Study subject number in each unit

\begin{tabular}{|c|c|}
\hline Unit & Number \\
\hline Peking University People's Hospital & 103 \\
\hline Guangdong Second Provincial General Hospital & 42 \\
\hline Chinese People's Liberation Army General Hospital & 48 \\
\hline Third Affiliated Hospital of Southern Medical University & 82 \\
\hline Beijing Jishuitan Hospital & 49 \\
\hline China-Japan Union Hospital of Jilin University & 66 \\
\hline $\begin{array}{l}\text { The First Affiliated Hospital of Guangxi University of } \\
\text { Chinese Medicine }\end{array}$ & 56 \\
\hline Peking University Third Hospital & 19 \\
\hline The Second Hospital of Anhui Medical University & 43 \\
\hline Sun Yat-Sen Memorial Hospital & 47 \\
\hline Fujian Provincial Hospital & 38 \\
\hline $\begin{array}{l}\text { The First Hospital Affiliated to Army Medical University } \\
\text { (Southwest Hospital) }\end{array}$ & 24 \\
\hline $\begin{array}{l}\text { Shanghai Jiao Tong University Affiliated First People's } \\
\text { Hospital }\end{array}$ & 18 \\
\hline Foshan Hospital of Traditional Chinese Medicine & 44 \\
\hline $\begin{array}{l}\text { Shanghai Jiao Tong University Affiliated Sixth People's } \\
\text { Hospital }\end{array}$ & 25 \\
\hline The First Affiliated Hospital of Fujian Medical University & 21 \\
\hline Haikou People's Hospital & 11 \\
\hline $\begin{array}{l}\text { Shanghai Guanghua Hospital of Integrated Traditional } \\
\text { Chinese and Western Medicine }\end{array}$ & 19 \\
\hline $\begin{array}{l}\text { The First Affiliated Hospital of University of Science } \\
\text { and Technology of China }\end{array}$ & 11 \\
\hline $\begin{array}{l}\text { Central Hospital Affiliated to Shen Yang Medical } \\
\text { College }\end{array}$ & 18 \\
\hline Zhongda Hospital Southeast University & 22 \\
\hline Yan'an Hospital of Kunming City & 26 \\
\hline Sichuan University West China Hospital & 51 \\
\hline $\begin{array}{l}\text { Luoyang Orthopedic-Traumatological Hospital of } \\
\text { Henan Province }\end{array}$ & 7 \\
\hline Jiangsu Province Hospital & 2 \\
\hline $\begin{array}{l}\text { Second Affiliated Hospital of Chongqing Medical } \\
\text { University }\end{array}$ & 2 \\
\hline $\begin{array}{l}\text { The Second Affiliated Hospital of Harbin Medical } \\
\text { University }\end{array}$ & 6 \\
\hline Total & 900 \\
\hline
\end{tabular}

